ARTICULOS ORIGINALES Rev Chil Salud Pública 2013

Vol 17 (1): 40-47

\section{Evaluación de conocimientos \\ en comunidades intervenidas por el "Programa de Promoción y Prevención en Salud Bucal para Preescolares" Región Metropolitana}

\section{RESUMEN}

En Chile la caries afecta a gran parte de la población, comprometiendo su calidad de vida desde temprana edad, particularmente en los sectores más vulnerables de la sociedad. La Educación para la salud es la estrategia que utiliza el "Programa de Promoción y Prevención en Salud Bucal para Preescolares" para motivar e incorporar hábitos saludables en preescolares, sus padres y funcionarios a cargo de su cuidado.

Este estudio descriptivo, tuvo por objetivo determinar el nivel de conocimientos en Salud Bucal de funcionarios y apoderados de jardines JUNJI e INTEGRA, según su participación en talleres educativos del programa durante 2010, para lo cual se realizó un test tipo encuesta autoaplicada. Se obtuvo una muestra de 558 encuestas válidas (261 funcionarios y 297 apoderados).

Resultados: Un 56\% los de funcionarios y un 22,8\% de los apoderados encuestados declaró haber participado en los talleres de salud bucal realizados en los jardines infantiles. Los funcionarios y apoderados capacitados en los talleres educativos presentaron un mayor porcentaje de aprobación del test de conocimientos en salud bucal respecto que sus pares no capacitados.

Conclusión: La capacitación mediante talleres educativos en salud bucal mejora sustancialmente los conocimientos en funcionarios y apoderados. Con ello, aportando a los objetivos del programa de Promoción y Prevención en Salud Bucal para Preescolares.

Palabras clave: Promoción de Salud, Determinantes Sociales de la Salud, Preescolares.

Víctor ReVello'; MACAReNa IsLeR ${ }^{2}$.

${ }^{1}$ Cirujano Dentista. Unidad de Salud Bucal- SEREMI de Salud, Región Metropolitana ${ }^{2}$ Cirujano Dentista, Universidad Nacional Andrés Bello.

TRABAJO DE INVESTIGACIÓN. Departamento de Salud Pública,

Facultad de Odontología, Universidad Nacional Andrés Bello, Santiago - Chile. 2011 victor.revello@redsalud.gov.cl

\section{KNOWLEDGE EVALUATION WITHIN COMMUNITIES THAT PARTICIPATE in the Oral Health Promotion and Prevention Program for Preschoolers in the Metropolitan Region}

\begin{abstract}
In Chile, dental caries affect a large part of the population, compromising their quality of life from an early age, particularly in the most vulnerable sectors of society. The Oral Health Promotion and Prevention Program uses a health education strategy in order to motivate healthy habits in preschoolers, their parents, and childcare workers. The objective of this descriptive study was to determine oral health knowledge in childcare workers and parents
\end{abstract}


and guardians in JUNJI and INTEGRA preschools, according to their participation in educational workshops during 2010. A self-administered survey was used in a sample of 558 participants (261 childcare workers and 297 parents and guardians).

Results: $56 \%$ of childcare workers and $22.8 \%$ of parents and guardians had participated in oral health workshops offered in preschools. A greater percentage of those who were trained in these workshops passed the knowledge test, compared to their peers who were not trained.

Conclusion: Oral health educational workshops substantially increase the knowledge of childcare workers and parents and guardians, and should contribute to reaching the goals of the Oral Health Promotion and Prevention Program for Preschoolers.

Key words: Health promotion, social determinants of health, preschoolers

\section{INTRODUCCIÓN}

La Salud contempla una relación de equilibrio que es posible, entre otros aspectos, con un estado bucal favorable. La situación epidemiológica de las patologías bucales en Chile muestra una alta prevalencia y severidad, pero además una distribución en la población marcada por los determinantes sociales, donde el daño se concentra en los grupos más vulnerables de nuestra sociedad. La caries dental se inicia desde los primeros años de vida y aumenta con la edad. Estudios nacionales indican que el 17\% de los niños y niñas de 2 años ya tiene caries, aumentando a un $50 \%$ a los 4 años y a un $70 \%$ a los 6 años, así como también aumenta dramáticamente el promedio de dientes afectados por caries (severidad), especialmente de los 2 a los 4 años donde aumenta casi 5 veces ${ }^{1}$.

Una revisión sistemática ${ }^{2}$ efectuada el año 1999 respecto al estado socioeconómico y determinantes del desarrollo como factores de riesgo de caries, concluyó que existía una fuerte evidencia de una relación inversa entre estado socioeconómico y la prevalencia de caries en niños menores de 12 años. Esta problemática influye en la calidad de vida de los individuos, vulnerando la autoestima, dificultando las relaciones sociales y los potenciales de éxito personal.

La prevención desde temprana edad es la manera más efectiva de evitar la aparición de problemas odontológicos, incorporando hábitos y costumbres saludables en el hogar, jardín infantil y escuela ${ }^{3}$. Para motivar y educar a los niños en salud bucal, se debe primero disponer de conocimientos adecuados y suficientes en los padres y agentes educativos de los establecimientos educacionales, quienes son los en- cargados de la estimulación temprana de los niños y gestores de sus primeros hábitos.

El Programa de Promoción y Prevención en Salud Bucal para Preescolares es una iniciativa que tiene por objetivo disminuir el daño prematuro por caries en preescolares menores de 6 años, mediante instalación de hábitos saludables de higiene bucal y alimentación, para lo cual se hace entrega de cepillos y pastas dentales, se realizan talleres educativos en alimentación saludable y técnicas de cepillado en los jardines infantiles para los preescolares, sus padres y agentes educativos. Además, se promueve acceso controlado y supervisado a fluoruros desde los 2 años, por medio de aplicación profesional barniz de flúor y uso de pasta dental fluorada de 500 ppm bajo control de un adulto ${ }^{4}$. Este conjunto de acciones sistemáticas, que tienen por objetivo mantener a la población preescolar libre de caries, se inició el año 2007 en 2 comunas de la región Metropolitana y en la actualidad beneficia 81 comunas a lo largo de todo el país, de las cuales 10 están en la Región Metropolitana: Cerro Navia, Colina, El Monte, La Pintana, Lampa, Maipú, María Pinto, Padre Hurtado, San Ramón y Til Tils.

En la actualidad no se dispone de información publicada sobre la evaluación de este programa, por lo que su desarrollo y expansión futura requiere de antecedentes respecto del logro de sus objetivos. Esta investigación pretende determinar el nivel de conocimientos en Salud Bucal de funcionarios y apoderados de jardines JUNJI e INTEGRA, según su participación en los talleres educativos del Programa Promoción y Prevención en Salud Bucal para preescolares en la Región Metropolitana durante el año 2010. 


\section{MATERIALES Y MÉTODOS}

\section{Diseño: Estudio descriptivo}

La población objetivo del estudio son: Padres, apoderados y funcionarios de jardines infantiles pertenecientes a Fundación INTEGRA y JUNJI de las 10 comunas intervenidas en la región Metropolitana que forman parte del "Programa de Promoción y Prevención en Salud Bucal de preescolares" durante el año 2010. La selección de la muestra fue no probabilístico por conveniencia, distribuida en jardines infantiles según la aplicación de los siguientes criterios de inclusión:

- Existencia de antecedentes disponibles y verificables en la SEREMI de Salud sobre la realización de talleres educativos en el jardín tanto para padres, como para funcionarios durante el año 2010. Se considera Taller Educativo en Salud Bucal, a la realización de una actividad programada en las dependencias del jardín, por parte de los equipos odontológicos de atención primaria, consistente en una jornada educativa con una duración mínima de 2 horas pedagógicas, en la cual se entregaron conocimientos en las siguientes áreas: alimentación saludable, alimentos cariogénicos, uso de pasta con flúor, consumo de agua potable, acceso a atención dental en preescolares y técnica de cepillado.

- Número de matrícula en Nivel Medio Mayor intervenidos sobre 25 niños y niñas.

- Existencia de número de total de funcionarios conocidos y declarados por jardín.

Para la medición del conocimiento por parte de funcionarios y apoderados se utilizaron 2 test tipo encuestas anónimas autoaplicadas. La encuesta de Funcionarios (Anexo $\mathrm{N}^{\circ} 1$ ) contaba con un total de 15 preguntas, mientras que la de Padres y Apoderados (Anexo $\mathrm{N}^{\circ} 2$ ) contaba con 16 preguntas. Las encuestas presentaban preguntas de selección múltiple, con una única respuesta correcta. Cada respuesta correcta equivale a un punto, y el puntaje total es la suma de todas las respuestas correctas. No se aplicó descuentos por omitidas o incorrectas. Se clasificó como aprobado el test, con un nivel de exigencia del $60 \%$, que equivalen a 9 puntos en el caso de la encuesta de 15 preguntas de funcionarios, y 10 puntos en la encuesta de 16 preguntas de apoderados.

Las preguntas de la encuesta estaban validadas por la Encuesta de Saberes y Creencias en Salud Bucal que utilizó el Ministerio de Salud el año 2009 para la evaluación de la Reformulación del Programa Odontológico en las comunas de Olmué (V Región, Paredones (VI Región) y El Monte (R.M.). Instrumento de evaluación tipo test de 41 preguntas abiertas y cerradas, disponible en formato papel en el Departamento Salud Bucal de la Subsecretaria Ministerial de Salud Pública MINSAL.

La encuesta se distribuyó por funcionarios de la SEREMI de Salud R.M. en cada jardín seleccionado, durante el mes de octubre de 2010 y recolectadas en diciembre de 2010 . Se distribuyeron un total de 394 encuestas para funcionarios y 434 encuestas para apoderados. En la recolección de las encuestas se obtuvieron un total de 558 encuestas (261 funcionarios y 297 apoderados), se presentó una pérdida del $32,7 \%$ de las encuestas (33,8\% en funcionarios, $31,6 \%$ en apoderados). Para la realización de este estudio se contó con la autorización de las direcciones regionales de JUNJI e INTEGRA. Este estudio fue aprobado por el comité de Investigación de la Universidad Andrés Bello.

Variables de investigación:

a) Tipo de encuestado (Funcionario - Apoderado)

b) Aprobación en las Encuestas (Aprobado - No aprobado)

c) Capacitación en Talleres de Salud Bucal (Capacitado - No capacitado)

d) Dependencia jardín (Junji - Integra)

\section{RESULTADOS}

Para analizar y obtener conclusiones a partir de los datos obtenidos según los objetivos planteados para esta investigación, se realizaron test estadísticos Z, Chi-cuadrado y análisis de correspondencias múltiples, usando para todos un valor de significancia estadística del 95\% (p 0,05).

En la Tabla 1 se puede observar la muestra del estudio clasificada por funcionarios y apoderados de acuerdo a su pertenencia a JUNJI o INTEGRA. 


\section{Anexo $\mathrm{N}^{\circ} 1$}

Test de conocimientos básicos en salud bucal para funcionarios de jardines infantiles comuna

\begin{tabular}{|l|l|}
\hline NOMBRE JARDIN & DEPENDENCIA \\
\hline CARGO & EDAD \\
\hline
\end{tabular}

\section{MARQUE SOLO UNA ALTERNATIVA}

1. Usted ha participado en los Talleres de Capacitación en Salud Bucal que se han desarrollado en el jardín infantil: a) Sí

b) No

2. ¿Cuál es el cepillado más importante del día, que NUNCA debe olvidar de realizar el niño(a)?

a) En la mańana al levantarse o después del desayuno

b) Antes de acostarse a dormir

c) Después de almorzar

d) Después de colación de la tarde

e) Después de comer un dulce

3. ¿Qué riesgos se corren si se pierde un diente de leche por caries?

a) Que el niño(a) sea víctima de burlas por sus compañeros

b) Los dientes definitivos podrían salir chuecos y feos

c) Podría tener dificultades para hablar

d) Podría infectar al diente definitivo que viene en camino

e) Todas las anteriores

4. ¿En qué alimentos se encuentran los hidratos de carbono que desarrollan las caries?

a) En las jaleas y yogurt

b) En las alimentos ricos en harina refinada como el queque, las galletas y los pasteles

c) En los azúcares de las bebidas y jugos artificiales

d) En los alimentos ricos en masas como el pan y los tallarines

e) Todos los anteriores

5. ¿Qué tipo de alimentos NO producen caries?

a) Las bebidas y jugos artificiales

b) Las frutas y verduras

c) Las masas dulces como las galletas

d) Los dulces como los caramelos y las pastillas de menta

e) El pan con queso

6. ¿Qué Programas Odontológicos están disponibles para los niños y niñas en el Consultorio?

a) Programa Odontológicos para niños(as) de 2 años de edad

b) Programa Odontológicos para niños(as) de 4 años de edad

c) Control Odontológico del Niño(a) Sano

d) Atención de Urgencia Dental

e) Todos los anteriores

7. A qué edad se debe iniciar el cepillado CON pasta de dientes infantil en los niños y niñas:

a) $A$ los 6 años

b) A los 2 años

c) $\mathrm{A}$ los 4 años

d) $\mathrm{A}$ los 5 años

e) $\mathrm{A} \operatorname{los} 3$ años

8. ¿Cuáles son las características de un cepillo adecuado para la higiene en los niños y niñas:

a) Cabeza grande, cerdas suaves, mango curvo anatómico y debe cambiarse idealmente una vez al año

b) Cabeza pequeña, cerdas suaves, mango recto anatómico y debe cambiarse idealmente cada 4 meses

c) Cabeza pequeña, cerdas duras, mango recto anatómico y debe cambiarse idealmente dos veces al año

d) No lo sé

9. ¿Cuáles de los siguientes elementos o situaciones pueden ocasionar malformaciones en la boca de un niño, si las realiza por mucho tiempo?

a) El chupete

b) La mamadera

c) Chuparse el dedo

d) Morderse las uñas

e) Todos los anteriores

10. Respecto de las siguientes afirmaciones, marque la FALSA:

a) El uso excesivo y sin control médico de antibióticos mancha los dientes y los debilita

b) El embarazo ocasiona pérdida de dientes

c) La mamadera de la noche ocasiona graves caries en los niños y niñas

d) Las caries se contagian de una boca a otra

e) El consumo de leche ayuda a proteger los dientes

11. Cuáles de los siguientes métodos de cuidado de la salud bucal es el más efectivo y beneficioso?

a) El uso de enjuagatorios bucales

b) El uso de seda dental

c) La visita al dentista

d) El cepillado frecuente con pasta dental con flúor

12. ¿A qué edad se debe llevar por primera vez al dentista a los niños/niñas?

a) A los 6 años edad

b) A los 4 años edad

c) Recién nacido

d) A los 2 años de edad

13. ¿En cuál de los siguientes elementos encontramos flúor para proteger los dientes?

a) Agua potable

b) Bebidas y jugos naturales

c) Yogurt

d) Frutas y verduras

14. Las pastas dentales infantiles para niños y niñas entre los 2 y 6 años en la etiqueta deben señalar la siguiente concentración de flúor

a) $1.400 \mathrm{ppm}$ de flúor

b) $500 \mathrm{ppm}$ de flúor

c) $100 \mathrm{ppm}$ de flúor

d) $1.000 \mathrm{ppm}$ de flúor

15. ¿Cuáles de los siguientes métodos de cuidado de la salud bucal es el más efectivo y beneficioso?

a) El uso de enjuagatorios bucales

b) El uso de seda dental

c) La visita al dentista

d) El cepillado frecuente con pasta dental 
Anexo $\mathrm{N}^{\circ} 2$

Test de conocimientos básicos en salud bucal para padres y apoderados de preescolares comuna

\begin{tabular}{|l|l|l|l|l|l|}
\hline NOMBRE JARDIN & & & DEPENDENCIA & JUNJI \\
\hline EDAD DEL MENOR & años & meses & PARENTESCO APODERADO Madre & $\begin{array}{l}\text { Hermano(a) } \\
\text { Otros }\end{array}$ \\
\hline
\end{tabular}

MARQUE SOLO UNA ALTERNATIVA

1. ¿Usted ha participado en los Talleres de Educación en Salud Bucal que se han desarrollado en el jardín infantil?

a) Sí

b) No

2. ¿Cuál es el cepillado más importante del día, que NUNCA debe olvidar de realizar mi hijo(a)?

a) En la mañana al levantarse o después del desayuno

b) Antes de acostarse a dormir

c) Después de almorzar

d) Después de colación de la tarde

e) Después de comer un dulce

3. ¿Qué integrante de la familia del niño(a) tiene más probabilidades de contagiarlo con las bacterias de las caries?

a) El papá

b) La abuela

c) La mamá

d) Los hermanos

e) No sé

4. ¿Qué riesgos se corren si se pierde un diente de leche por caries?

a) Que el niño(a) sea víctima de burlas por sus compañeros

b) Los dientes definitivos podrían salir chuecos y feos

c) Podría tener dificultades para hablar, ya que los dientes participan en la fonación

d) Podría infectar al diente definitivo que viene en camino

e) Todas las anteriores

5. ¿En qué alimentos se encuentran los hidratos de carbono que desarrollan las caries?

a) En las jaleas y yogurt

b) En las alimentos ricos en harina refinada como el queque, las galletas y los pasteles

c) En los azúcares de las bebidas y jugos

d) En los alimentos ricos en masas como el pan y los tallarines

e) Todos los anteriores

6. ¿Qué tipo de alimentos NO producen caries?

a) Las bebidas y jugos artificiales

b) Las frutas y verduras

c) Las masas dulces como las galletas

d) Los dulces como los caramelos y pastillas de menta

e) El pan con queso

7. ¿Qué Programas Odontológicos están disponibles para los niños y niñas en el Consultorio?

a) Programa Odontológicos para niños(as) de 2 años de edad

b) Programa Odontológicos para niños(as) de 4 años de edad

c) Control Odontológico del Niño(a) Sano

d) Atención de Urgencia Dental

e) Todos los anteriores

8. ¿A qué edad se debe iniciar el cepillado CON pasta de dientes infantil en los nuestros hijos(as)?

a) $\mathrm{A} \operatorname{los} 6$ años

b) $\mathrm{A} \operatorname{los} 2$ años

c) $\mathrm{A} \operatorname{los} 4$ años

d) $\mathrm{A}$ los 5 años

e) $\mathrm{A} \operatorname{los} 3$ años

9. ¿Cuáles son las características de un cepillo adecuado para la higiene en los niños y niñas: a) Cabeza grande, cerdas suaves, mango curvo anatómico y debe cambiarse idealmente una vez al año

b) Cabeza pequeña, cerdas suaves, mango recto anatómico y debe cambiarse idealmente cada 4 meses

c) Cabeza pequeña, cerdas duras, mango recto anatómico y debe cambiarse idealmente dos veces al año

d) No lo sé

10. Cuáles de los siguientes elementos o situaciones pueden ocasionar malformaciones en la boca de un niño, si las realiza por mucho tiempo?

a) El chupete

b) La mamadera

c) Chuparse los dedos

d) Morderse las uñas

e) Todos los anteriores

11. Respecto de las siguientes afirmaciones, marque la FALSA:

a) El uso excesivo y sin control médico de antibióticos mancha los dientes y los debilita

b) El embarazo ocasiona pérdida de dientes

c) La mamadera de la noche ocasiona graves caries en los niños y niñas

d) Las caries se contagian de una boca a otra

e) El consumo de leche ayuda a proteger los dientes

12. ¿Cuáles de los siguientes métodos de cuidado de la salud bucal es el más efectivo y beneficioso?

a) El uso de enjuagatorios bucales

b) El uso de seda dental

c) La visita al dentista

d) El cepillado frecuente con pasta dental con flúor

13. ¿A qué edad debemos llevar por primera vez al dentista a nuestros hijos(as)?

a) A los 6 años edad

b) A los 4 años edad

c) Recién nacido

d) A los 2 años de edad

14. ¿En cuál de los siguientes elementos encontramos flúor para proteger los dientes?:

a) Agua potable

b) Bebidas y jugos naturales

c) Yogurt

d) Frutas y verduras

e) En ninguno de estos elementos

15. Las pastas dentales infantiles en la etiqueta deben señalar la siguiente concentración de flúor

a) $1.400 \mathrm{ppm}$ de flúor

b) 500 ppm de flúor

c) $100 \mathrm{ppm}$ de flúor

d) $1.000 \mathrm{ppm}$ de flúor

16. ¿A qué edad su hijo(a) tendrá derecho al GES Odontológico en el consultorio?

a) A los 2 años

b) A los 4 años

c) A los 6 años

d) A los 12 años

e) No sé cuando 
Tabla 1. Muestra total del estudio según tipo de encuestado y dependencia

\begin{tabular}{|lccc|}
\hline & \multicolumn{2}{c}{ Dependencia } & Total \\
& JUNJI & INTEGRA & \\
\hline Funcionarios & 128 & 118 & 246 \\
Apoderados & 163 & 126 & 289 \\
\hline
\end{tabular}

En las Tablas 2 y 3 se puede observar la muestra del estudio clasificada por funcionarios y apoderados según su participación en talleres educativos ajustado por dependencia. En el caso de los funcionarios, del total de encuestados el $56 \%$ declaró haber participado en talleres de capacitación, y en el caso de los apoderados sólo el 22,8\% declaró haberlo realizado.

Tabla 2. Total de funcionarios según capacitación en talleres de Salud Bucal y dependencia

\begin{tabular}{|lccc|}
\hline \multicolumn{3}{c}{ Funcionarios } & Total \\
& Capacitados & No Capacitados \\
\hline JUNJI & $62(48 \%)$ & $66(52 \%)$ & $128(100 \%)$ \\
INTEGRA & $76(64 \%)$ & $42(36 \%)$ & $118(100 \%)$ \\
Totales & $138(56 \%)$ & $108(44 \%)$ & $246(100 \%)$ \\
\hline
\end{tabular}

Tabla 3. Total de apoderados según capacitación en talleres de Salud Bucal y dependencia

\begin{tabular}{|lccc|}
\hline \multicolumn{3}{c}{ Apoderados } & Total \\
& Capacitados & No Capacitados \\
\hline JUNJI & $46(28 \%)$ & $117(72 \%)$ & $163(100 \%)$ \\
INTEGRA & $20(16 \%)$ & $106(84 \%)$ & $126(100 \%)$ \\
Totales & $66(22,8 \%)$ & $223(77,2 \%)$ & $289(100 \%)$ \\
\hline
\end{tabular}

En la Tabla 4 se observa los resultados de aprobación obtenidos por los funcionarios, la cual señala que el porcentaje de funcionarios aprobados capacitados $(72,5 \%)$ resultó significativamente superior que el porcentaje de funcionarios aprobados no capacitados en los talleres (59,3\%), con $\mathrm{p}<0,05$.

Tabla 4. Total de funcionarios según aprobación y capacitación en talleres de Salud Bucal

\begin{tabular}{|lcccc|}
\hline & \multicolumn{4}{c|}{ Funcionarios } \\
& Capacitados & No capacitados \\
& $\mathrm{n}$ & $\%$ & $\mathrm{n}$ & $\%$ \\
\hline Reprobado & 38 & $27,50 \%$ & 44 & $40,70 \%$ \\
Aprobado & 100 & $72,50 \%$ & 64 & $59,30 \%$ \\
Totales & 138 & $100,00 \%$ & 108 & $100,00 \%$ \\
\hline
\end{tabular}

Realizando el análisis desagregado por la variable de ajuste dependencia del jardín (JUNJI-INTEGRA), no se detectan diferencias significativas el porcentaje de aprobación del test de conocimientos entre funcionarios capacitados y no capacitados.

En la Tabla 5 se observa el resultado del test a nivel de padres y apoderados, la cual señala que el porcentaje de apoderados capacitados que aprobaron el test $(71,2 \%)$ es significativamente superior que los apoderados no capacitados (32,3\%), con un $\mathrm{p}<0,05$. Desagregando por la variable dependencia del jardín (JUNJI-INTEGRA), se mantiene la tendencia de mayor nivel de aprobación en los apoderados capacitados.

Tabla 5. Total de apoderados según aprobación y capacitación en talleres de Salud Bucal

\begin{tabular}{|c|c|c|c|c|}
\hline & \multicolumn{4}{|c|}{ Apoderados } \\
\hline & \multicolumn{2}{|c|}{ Capacitados } & \multicolumn{2}{|c|}{ No Capacitados } \\
\hline & $\mathrm{n}$ & $\%$ & $\mathrm{n}$ & $\%$ \\
\hline Reprobado & 19 & $28,80 \%$ & 151 & $67,70 \%$ \\
\hline Aprobado & 47 & $71,20 \%$ & 72 & $32,30 \%$ \\
\hline Totales & 66 & $100,00 \%$ & 223 & $100,00 \%$ \\
\hline
\end{tabular}

Para finalizar el análisis, se realizó un gráfico de correspondencias múltiples (ver Gráfico $\mathrm{N}^{\circ} 1$ ), el cual permite observar una fuerte tendencia en la muestra entre ser apoderado, no estar capacitado y reprobar el test. A nivel de funcionarios, la tendencia es ser capacitado y aprobar el test de conocimientos. Señala además que, la variable de dependencia del jardín, ya sea JUNJI o INTEGRA no es una condicionante importante en los resultados a nivel de apoderados y funcionarios.

\section{DISCUSIÓN Y CONCLUSIONES}

En el marco del programa de Promoción y Prevención en Salud Bucal para Preescolares, se realizó una investigación basal en el año 2009 que tenía por objetivo evaluar el nivel de conocimientos en salud bucal de la comunidad educativa de 64 jardines infantiles de 4 comunas vulnerables intervenidas por el programa en la Región Metropolitana ${ }^{6}$, el cual concluyó que el nivel de conocimientos para el cuidado de salud bucal de la comunidad educativa intervenida por el programa es bajo, obteniendo un porcentaje de aprobación 


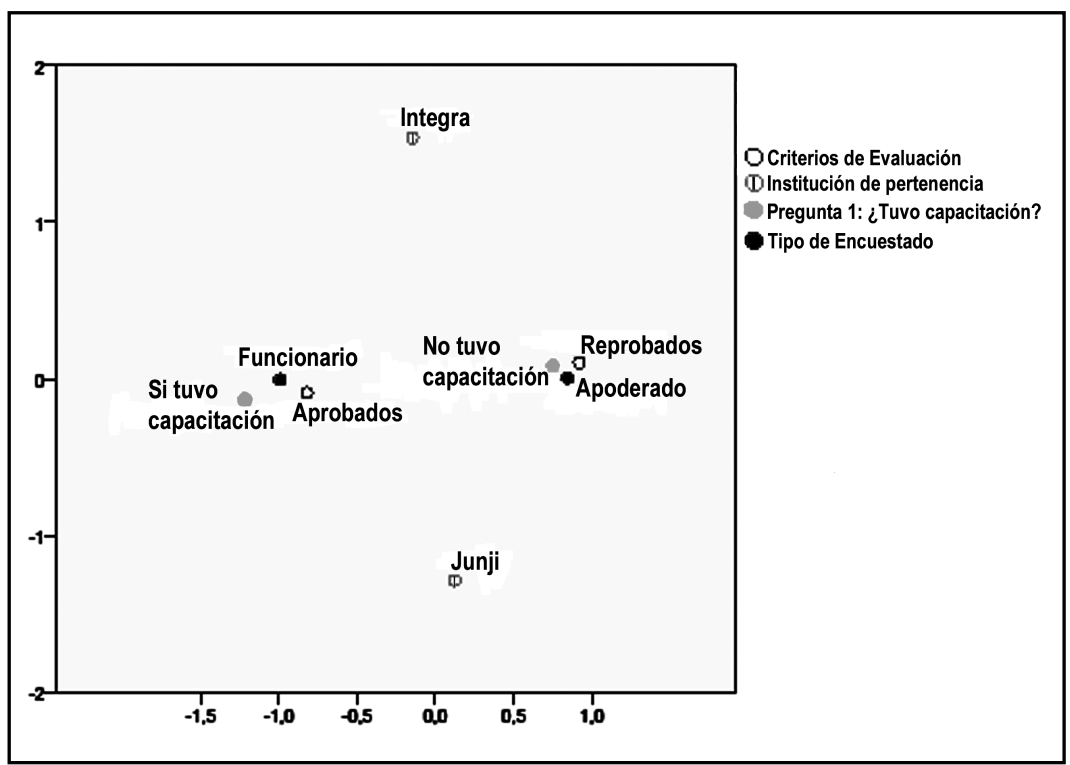

Gráfico $\mathrm{N}^{\circ} 1$. Análisis de correspondencias múltiples entre Tipo de Encuestado, Aprobación del Test, Capacitación y Dependencia del jardín

del test de un $26 \%$ en el total de los encuestados. En una visión general, el presente estudio observó que estas cifras mejoraron respecto de ese estudio, obteniendo un porcentaje de aprobación en funcionarios de $59,1 \%$ y en apoderados $40,1 \%$. No obstante, estos datos son sólo referenciales ya que el instrumento utilizado (test) fue modificado para este estudio.

Este programa no presenta evaluaciones de ningún tipo publicadas al año 2010, este estudio es la primera evaluación en uno de sus componentes, específicamente en el nivel de conocimientos en salud bucal de padres y funcionarios a cargo del cuidado de los preescolares beneficiarios. El análisis por tipo de encuestado, nos señala que en caso de los funcionarios, el $56,09 \%$ de los encuestados declaró haber participado en los talleres educativos del programa, comparado este nivel de participación con el de los padres, es bastante alto, atribuible al hecho de realizarse los talleres principalmente en el mismo jardín, y estar este programa parcialmente institucionalizado en JUNJI e INTEGRA, lo cual facilita la integración entre los equipos odontológicos de atención primaria y los agentes educativos de los jardines. Los talleres educativos presentaron un impacto positivo en los funcionarios, dado que los funcionarios capacitados presentaron un mayor porcentaje de aprobación $(72,5 \%)$ que sus pares no capacitados (59\%). Si bien, resultó ser una diferencia estadísticamente significativa en general, si el análisis se realiza desagregado por dependencia (JUNJI-INTEGRA), la diferencia estadística significativa entre porcentaje de funcionarios capacitados aprobados y no capacitados aprobados desaparece, hecho atribuible principalmente a la reducción de los tamaños muestrales. Es importante la consideración de la dependencia técnica-administrativa de los jardines, ya que presentan diferencias destacables tales como: los jardines INTEGRA habitualmente disponen de solo una profesional Educadora de Párvulos a cargo del jardín y técnicos a cargo del cuidado directo de los párvulos en la sala de clase, en el caso de JUNJI, existe una mayor dotación de profesionales Educadoras de Párvulos por jardín, apoyadas por Técnicos Parvularias, también existiría en base a la observación en terreno y cálculo de dotaciones de funcionarios un mayor número promedio de párvulos por agente educativo en los jardines INTEGRA, respecto de JUNJI. En resumen, en el caso de los funcionarios, hay un mayor acceso a las capacitaciones y existiría una preparación básica en gran parte de los funcionarios respecto a salud bucal y enseñanza de hábitos saludables en niños preescolares, lo cual sería mejorable mediante su participación en los talleres de capacitación del programa. 
Por otro lado, en los apoderados se observa una menor participación en los talleres de capacitación, siendo ésta más baja en los jardines INTEGRA con un $16 \%$ de los encuestados y un $28 \%$ en los jardines JUNJI. Este bajo nivel de participación de los apoderados sería atribuible a los topes horarios con actividades laborales de los padres, falta de interés, falta de información de la realización de la actividad por parte del jardín a los apoderados de los párvulos u otros factores sociales que interés de investigar en futuras investigaciones. En el caso de los apoderados, se observó una gran diferencia entre el porcentaje de apoderados capacitados aprobados $(71,2 \%)$, respecto de los aprobados no capacitados $(32,3 \%)$, la cual es estadísticamente significativa incluso desagregando por dependencia del jardín (JUNJI-INTEGRA). A partir de estos datos, se concluye que para los padres y apoderados es altamente significativo capacitarse para mejorar su nivel de información en salud bucal, más que para los funcionarios. Sin embargo, existirían barreras que impiden su mayor participación en los talleres de capacitación que se desarrollan en el marco del programa de Promoción y Prevención en Salud Bucal para Preescolares. Esto motiva a seguir investigando en las barreras y su superación, así como en mejorar la calidad de los talleres educativos.

En la búsqueda bibliográfica realizada para este estudio, no se encontraron estudios similares de evaluación de metodologías de educación o promoción en Salud Bucal, los cuales hubiesen sido interesantes de comparar con resultados obtenidos en esta investigación. Sin embargo, dado los antecedentes de esta investigación, específicamente del grupo de apoderados, se establece la importancia de estos talleres como un medio de educación e incentivo esencial para la adopción de conductas saludables e incrementar y fortalecer los conocimientos en salud bucal, tanto en niños como adultos.

\section{REFERENCIAS}

1. Boletín N 1 "Dientes Sanos", Programa de Promoción y Prevención de Salud Bucal para preescolares. Ministerio de Salud, Gobierno de Chile, Septiembre de 2010. [En Internet]. Disponible en: http:// w w w. in tegra.cl/integra.nsf/data/ 629178F16ED0CEDB842577D00068A3B1/\$File/ Boletin\%20ODONTOLOGICOn\%C2\%BA1.pdf [Accesado el 25 de Junio de 2011].

2. Watt R, Sheihan A. Inequality in oral Health: a review of evidence and recommendation for action. Br Dent J 1999; 187(1): pp 6-12.

3. Diéguez E et al. (2002). Educación Dental Infantil: Guía para profesionales de educación y de salud (Segunda Edición Revisada) [En Internet]. Editado por la Junta de Andalucía, Consejería de Salud, 2002, Sevilla, España. Disponible en: http://www.colombiaya.com/ lidavargas/documentos/manual-de-educacionbucal.pdf [Accesado el 22 de Junio de 2011].

4. Estrategia Nacional de Salud, para el cumplimiento de los Objetivos Sanitarios de la década 2011-2020. Santiago, Ministerio de Salud Gobierno de Chile. [En Internet]. Disponible en: http://www.minsal.gob.cl/portal/url/item/ b89e911085a830ace0400101650115af.pdf [Accesado el 25 de Junio de 2011].

5. "Programa de Promoción y Prevención en Salud Bucal de preescolares en la Región Metropolitana" (2007). [En Internet]. Secretaría Regional Ministerial de Salud, Departamento de Salud Pública, Gobierno de Chile. Disponible en: http:/ /www.redsalud.gov.cl/portal/url/item/ 7f2d789a972f153be04001011f012d29.pdf [Accesado el 22 de Junio de 2011].

6. Villegas MJ, Revello V. (2009). "Evaluación del nivel de conocimiento en Salud Bucal de la Comunidad Educativa de jardines infantiles de 4 comunas vulnerables de la Región Metropolitana”. Tesis Facultad de Odontología, Universidad Andrés Bello. 